\title{
The trend of radiological severity of hip fractures over a 30 years period: a cohort study
}

\author{
Mehdy Farhang, Sebastian Mukka, Ulrica Bergström, Olle Svensson and Arkan S. Sayed-Noor
}

\begin{abstract}
Background: Despite advances in operative techniques and preoperative care, proximal femur fractures (PFF) still represent a great public health problem. Displacement and fracture stability have been assumed as important determinants of treatment modality and outcome in such fractures. Purpose of this study was to determine whether the radiological severity of PFF fractures has increased over time.
\end{abstract}

Methods: In a cohort study, the plain radiographs of all patients with PFF aged over 50 years who were admitted to Umeå University Hospital in 1981/82, 2002 and 2012 were recruited to examine the types of fractures.

Results: The ratio of undisplaced to displaced femoral neck (FN) fractures was 30 to 70\% in 1981/82, 28 to $72 \%$ in 2002 and 25 to $75 \%$ in 2012. The ratio of stable to unstable intertrochanteric (IT) fractures was 64 to 36\% in 1981/ 82,68 to $32 \%$ in 2002 and 75 to $25 \%$ in 2012. The ratio of simple to comminute subtrochanteric fractures was 35 to $65 \%$ in 1981/82, 16 to $84 \%$ in 2002 and 12 to $88 \%$ in 2012. In both FN and IT fractures we found no statistical difference among these 3 study periods, $p=0.67$ and $p=0.40$. In subtrochanteric fractures we saw a tendency towards more comminute subtrochanteric fractures (1981/82 to 2012), $p=0.09$.

Conclusions: We found no significant increment in the radiological severity of FN and IT over a 30 years' period. However, there was tendency towards an increase in comminute subtrochanteric fractures.

Keywords: Hip fracture, Proximal femoral fracture, Trend, Severity

\section{Background}

Osteoporotic fractures and mainly those involving the proximal femur (PFF) represent a great public health problem, with a drastic economic burden on the community. In USA, nearly 300,000 PFF occur every year at a cost of over 8.8 billions USD while in Sweden, nearly 18,000 PFF occur every year at a cost of 200 millions USD [1-3]. Fractures of the femoral neck (FN) and the intertrochanteric (IT) region account for more than $90 \%$ of PFF and occur in approximately equal proportions. The incidence of these fractures seems to be flattening despite previous reports indicating a trend of increasing incidence as the population ages [4]. The mechanism of injury and risk factors for FN fractures differ from those associated with IT fractures and the FN fractures are more prevalent in people with impaired functional status

\footnotetext{
* Correspondence: arkansam@yahoo.com

Department of Surgical and Perioperative Sciences, Umeå University, 90187 Umeå, Sweden
}

and corticosteroid use while IT fractures occur in older and thinner patients with poor health status [5].

The functional outcome of PFF can be determined by several factors with variable influence. These factors include age, gender, preoperative morbidities, pre-fracture residence and ambulation, fracture type and functional status at hospital discharge [6,7]. Many of these patients never regain their pre-fracture walking ability and social independency. It is generally accepted that the displacement of FN fractures and the stability of IT fractures are important determinant of the treatment modality and outcome.

The purpose of this study was to review the radiological types of PFF treated at a single university hospital over a 30 years' period, to determine whether the severity of these fractures has increased with time. Our null hypothesis was that no such severity increment existed. 


\section{Methods}

\section{Study setting}

Umeå University Hospital is situated in the Northern part of Sweden and is responsible for all the emergency care for the city of Umeå and its surrounding neighbourhoods $\left(145,000\right.$ inhabitants and $\left.10,730 \mathrm{~km}^{2}\right)$. There is no other hospital in this part of Västerbotten County.

In retrospective cohort study, all patients with PFF (FN, IT and subtrochanteric fractures) aged over 50 years who were admitted to Umeå University Hospital during years 1980-2014 were included. Pathologic and periprosthetic fractures were excluded. The unique Swedish personal identification numbers were used.

\section{Data collection}

The database (UmanHip database) was compared with the hospital registers on an annual basis; thus, all in-hospital fractures were also registered. By crosschecking against the hospital's compulsory e-code registration regarding the reason for hospital admission, any possibility of losing in-patients in the data set was minimized. The following variables were registered: age, gender, side of fracture, fracture type (FN, IT or subtrochanteric, without grading), operation date, operative technique and reoperations.

The plain radiographs (antero-posterior and lateral views) of all PFF patients from 1981/82, 2002 and 2012 were recruited to examine the types of fracture. The FN fractures were classified as undisplaced (Garden 1 and 2 types) or displaced (Garden 3 and 4 types) [8], while the IT fractures were classified according to Evan [9] as stable (Simple non-comminute fractures) or unstable (with comminution of the calcar or lateral cortex with fragment larger than $5 \mathrm{~mm}$ ). Subtrochanteric fractures were defined as those whose main fracture line lay between the lesser trochanter and $5 \mathrm{~cm}$ distal to it, and classified according to Seinsheimer [10] as simple 2 fragments or comminute 3 or 4 fragments.

Radiographs from 1981/82 were available as analogue pictures while radiographs from 2002 and 2012 were available as digital pictures using PACS system.

To determine the interobserver and intraobserver reliability of the radiological measurements used in this study, a random set of digital radiographs $(n=244)$ were independently examined by two examiners, an orthopaedic surgeon and a radiologist, two times with 4-6 weeks' interval. The remaining radiographs $(n=412)$ were examined and classified by the orthopaedic surgeon.

\section{Statistical analysis}

The ages of the patients were compared using one-way analysis of variance (ANOVA) with post-hoc analysis. The distribution of gender and type of fracture was analysed with chisquared test. Interobserver and intraobserver reliability was studied using Cohen's Kappa Coefficient. We considered agreement as poor if $<0.2$, fair if between 0.21 and 0.40 , moderate if between 0.41 and 0.60 , substantial if between 0.61 and 0.80 and good if $>0.80$. A $p$-value $<0.05$ was considered to be statistically significant. Statistical analyses were performed using SPSS 17.0 (IBM, Armonk, New York).

\section{Results}

There were 7737 PFF [4342 FN fractures (56\%), 2772 IT fractures (36\%) and 623 subtrochanteric fractures (8\%)] admitted to Umeå University Hospital during the study period [nearly 30\% were men and 70\% were women, mean age of 81 (SD 9)]. The age-adjusted incidence per 100,000 and year was 799 in 1981/82, 637 in 2002 and 515 in 2012.

The Cohen's Kappa coefficient for interobserver reliability was 0.85 (95\% confidence interval $0.79-0.89$ ) and for the intraobserver reliability was 0.89 (95\% confidence interval 0.84-0.93).

There were 363 PFF fractures from 1981/82, of which analogue radiographs of 211 (58\%) PFF were available. There were 229 PFF fractures from 2002, of which digital radiographs of 218 (95\%) PFF were available while there were 235 PFF fractures from 2012, of which digital radiographs of 227 (97\%) PFF were available.

\section{The trend of radiological severity of PFF fractures}

In 1981/82, the number of undisplaced FN fractures was 36 (30\%) compared to 85 (70\%) displaced FN fractures. In 2002 the number of undisplaced FN fractures was 33 (28\%) compared to 86 (72\%) displaced FN fractures, while in 2012 the number of undisplaced FN fractures was 28 (25\%) compared to $86(75 \%)$ displaced FN fractures. We found no statistical difference among these 3 study periods, $p=0.67$ (Table 1 ).

In $1981 / 82$, the number of stable IT fractures was 47 (64\%) compared to 26 (36\%) unstable IT fractures. In 2002 the number of stable IT fractures was 38 (68\%) compared to 18 (32\%) unstable IT fractures, while in 2012 the number of stable IT fractures was 53 (75\%) compared to 18 (25\%) unstable IT fractures. We found no statistical difference among these 3 study periods, $p=0.40$ (Table 1 ).

In 1981/82, the number of simple subtrochanteric fractures was 6 (35\%) compared to $11(65 \%)$ comminute subtrochanteric fractures. In 2002, the number of simple subtrochanteric fractures was 7 (16\%) compared to 36 (84\%) comminute subtrochanteric fractures while in 2012, the number of simple subtrochanteric fractures was 5 (12\%) compared to 37 (88\%) comminute subtrochanteric fractures. There were a tendency for statistical difference among these 3 study periods, $p=0.09$ (Table 1 ).

\section{Discussion}

In this retrospective study, we found no significant increment in the radiological severity of FN and IT over a 30 years' period. However, there was a tendency towards an increase in comminute subtrochanteric fractures. 
Table 1 Results of the trend of radiological severity of hip fractures showing no differences over the study period

\begin{tabular}{lllll}
\hline & $1981 / 82$ & 2001 & 2012 & $\boldsymbol{p}$-value for difference among the 3 time periods \\
\hline Available radiographs & 211 & 218 & 227 & \\
Undisplaced FN fractures & $36(30 \%)$ & $33(28 \%)$ & $28(25 \%)$ & $p=0.67$ \\
Displaced FN fractures & $85(70 \%)$ & $86(72 \%)$ & $86(75 \%)$ & \\
Stable IT fractures & $47(64 \%)$ & $38(68 \%)$ & $53(75 \%)$ & $p=0.40$ \\
Unstable IT fractures & $26(36 \%)$ & $18(32 \%)$ & $18(25 \%)$ & \\
Simple subtrochanteric fractures & $6(35 \%)$ & $7(16 \%)$ & $5(12 \%)$ & $p=0.09$ \\
Comminuted subtrochanteric fractures & $11(65 \%)$ & $36(84 \%)$ & $37(88 \%)$ & \\
\hline
\end{tabular}

The bone composition of the proximal femur differs between the FN, IT and subtrochanteric regions and therefore it is possible that the etiology of the fractures in these different sites may also differ. The trochanteric region for instance has a greater proportion of trabecular bone compared with the FN and subtrochanteric regions [11]. Also, the geometric parameters such buckling ratio and hip axis length were more strongly linked with the severity type of PFF, although interaction terms were mostly not significant [12]. Worldwide, the recent trends in the incidence of PFF have varied widely: increase, plateau, or decrease. Epidemiological studies have shown that the incidence increases gradually with age, starting at 40 years, with a steep increase after 75 years of age $[4,13,14]$. The main underlying etiology is low bone mineral density (BMD) and increasing risk for falling.

Cauley et al. [5] studied the risk factors affecting the degree of severity of FN and IT fractures, determined by the degree of fracture displacement using the Garden's classification in FN fractures and the Kyle system for IT fractures, and found that displaced FN fractures were more common in older age, lower BMD, taller stature, corticosteroid use and poor functional status as measured by lower grip strength. On the other hand, Parkinson's disease and poor vision were associated with stable IT fractures. Chehade et al. [15] for instance found that unstable IT fractures were at greater odds of postoperative complications, reoperation and mortality within 6 and 12 months than those with stable fractures. On the other hand, Cornwell et al. [16] compared the functional outcomes and mortality among patients with different types of PFF classified as either non-displaced FN, displaced FN, or stable IT and unstable IT fractures. Despite that mortality was highest for displaced FN fracture patients and functional independence measure scores were least for unstable IT fracture patients compared with non-displaced FN fracture patients respectively, a multivariate analysis identified pre-injury age and function as predictors for mortality and functional outcome.

The results of this study concur with those reported by Lakstein et al. who also found no differences between the radiological severity of FN and IT fractures between 2001 and 2010 [17]. Only the IT fractures in patients older than
80 years of age showed increased proportion of unstable fractures. Contrary to this, Martínez et al. [18] found a significant increase in the incidence of displaced FN fractures and a decrease in the incidence of undisplaced FN fractures in women, while the incidence of different types of trochanteric fractures did not vary. Regarding subtrochanteric fractures, our results showed increased proportion and severity of comminuted fractures during the study period. The annual report of Swedish National Registry of hip fracture patient care in 2016 demonstrated a gradually increasing incidence of displaced FN and unstable IT fractures during the last three decades, both for the entire country and the geographical area of the present study population [19]. The treatment methods of these fractures have also changed towards the use of hip arthroplasty and intramedullary nail rather than screw fixation and sliding screw and plating, respectively. The report however does not include analyses whether these incidence and treatment method changes reach statistical significance. The variation of results reported in these studies is probably related to the geographical, time- and population-related factors affecting the fracture severity e.g. BMD, body mass index, medications and co-morbidities. In the present study, for instance, the mean age at hip fracture increased from 76.5 years in early 80 s to 81.5 years in 2012 . This could explain the tendency towards more comminuted fractures in older osteoporotic patients in later years. This is in contrast to the increasing consumption of anti-resorptive medications and corticosteroids in recent years. Other possible influencing factors include changing in injury panorama from high to low energy trauma and falls, smoking and alcohol habit. As the present study is a radiological analysis we have not included these factors in our evaluation. On the other side, there is no obvious reason for the increased comminution of subtrochanteric fractures in comparison to the IT counterparts. However, a gradually increasing lifespan, an age dependent inactivity and decrease in BMD might contribute to this finding.

This study has a number of limitations. First, it is a radiological analysis with no clinical parameters included. Factors like pre-fracture BMD, postoperative hospital stay length, complications and reoperations and mortality can 
reflect the fracture severity. Second, approximately $40 \%$ of 1981/82 radiographs were unavailable for analysis. However, we think this absence is random with no influence on the validity of the study results, but a selection bias cannot be ruled out which could explain the differences found in subtrochanteric fractures. Radiographs from 1981/82 were available as analogue form and therefore could not be blinded. This might have influenced the observers. However, the inter- and intraobserver reliability were very good. Third, our sample size is limited and we are not able to detect smaller differences in fracture pattern. Furthermore, the results can only be generalized to populations similar to those included in this study i.e. white elderly patients living in comparable socio-economic and environmental circumstances. These limitations are counterbalanced by the strengths of the study, which covers 30 years' data derived from a valid database on a stable population within a catchment area of a single center.

\section{Conclusions}

We found no significant increment of radiological fracture severity of FN and IT fractures over the study period while the subtrochanteric fractures showed a tendency for increased complexity with time. These results can assist caregivers to predict and plan the resources needed to manage these important fractures in the elderly population.

\section{Abbreviations}

ANOVA: Analysis of variance; FN: Femoral neck; IT: Intertrochanteric PACS: Picture archiving and communication system; PFF: Proximal femur fracture

\section{Acknowledgements}

Not applicable.

\section{Authors' contributions}

MF: study design, data collection and analysis, writing the manuscript. SM: study design, writing the manuscript. UB: study design, writing the manuscript. OS: study design, data analysis, writing the manuscript, supervision. ASN: study design, analysis, writing the manuscript, supervision. All authors have read and approved the final manuscript.

\section{Funding}

No specific funding was received.

\section{Availability of data and materials}

All data is stored in the trial registry. And the datasets used or analysed during the current study are available from the corresponding author on reasonable request.

\section{Ethics approval and consent to participate}

The study was conducted in accordance with the ethical principles of the Helsinki declaration and was approved by the regional Ethics Committee of Umeå University.

\section{Consent for publication}

N/A
Received: 15 January 2019 Accepted: 25 July 2019

Published online: 07 August 2019

References

1. Bentler SE, Liu L, Obrizan M, Cook EA, Wright KB, Geweke JF, Chrischilles EA, Pavlik CE, Wallace RB, Ohsfeldt RL, Jones MP, Rosenthal GE, Wolinsky FD. The aftermath of hip fracture: discharge placement, functional status change, and mortality. Am J Epidemiol. 2009;170:1290-9.

2. Dy CJ, McCollister KE, Lubarsky DA, Lane JM. An economic evaluation of a systems-based strategy to expedite surgical treatment of hip fractures. J Bone Joint Surg. 2011;93:1326-34.

3. Nyberg L, Gustafson Y, Berggren D, Brännström B, Bucht G. Falls leading to femoral neck fractures in lucid older people. J Am Geriatr Soc. 1996:44:156-60.

4. Michael Lewiecki E, Wright NC, Curtis JR, Siris E, Gagel RF, Saag KG, Singer AJ, Steven PM, Adler RA. Hip fracture trends in the United States, 2002 to 2015. Osteoporos Int. 2018;29(3):717-22.

5. Cauley JA, Lui LY, Genant HK, Salamone L, Browner W, Fink HA, Cohen P, Hillier T, Bauer DC, Cummings SR, Study of Osteoporotic Fractures Research and Group. Risk factors for severity and type of the hip fracture. J Bone Miner Res. 2009;24(5):943-55.

6. Liem IS, Kammerlander C, Suhm N, Blauth M, Roth T, Gosch M, Hoang-Kim A, Mendelson D, Zuckerman J, Leung F, Burton J, Moran C, Parker M, Giusti A, Pioli G, Goldhahn J, Kates SL. Investigation performed with the assistance of the AOTrauma network. Identifying a standard set of outcome parameters for the evaluation of orthogeriatric co-management for hip fractures. Injury. 2013:44(11):1403-12.

7. Kristensen MT. Factors affecting functional prognosis of patients with hip fracture. Eur J Phys Rehabil Med. 2011;47(2):257-64.

8. Van Embden D, Rhemrev SJ, Genelin F, Meylaerts SA, Roukema GR. The reliability of a simplified garden classification for intracapsular hip fractures. Orthop Traumatol Surg Res. 2012;98(4):405-8.

9. Andersen $\mathrm{E}$, Jørgensen LG, Hededam LT. Evans' classification of trochanteric fractures: an assessment of the interobserver and intraobserver reliability. Injury. 1990;21(6):377-8.

10. Seinsheimer F. Subtrochanteric fractures of the femur. J Bone Joint Surg Am. 1978;60(3):300-6.

11. Lu Y, Wang L, Hao Y, Wang Z, Wang M, Ge S. Analysis of trabecular distribution of the proximal femur in patients with fragility fractures. BMC Musculoskelet Disord. 2013;14:130.

12. Anitha $D$, Lee $T$. Assessing bone quality in terms of bone mineral density, buckling ratio and critical fracture load. J Bone Metab. 2014;21(4):243-7.

13. Amin S, Achenbach SJ, Atkinson EJ, Khosla S, Melton LJ 3rd. Trends in fracture incidence: a population-based study over 20 years. J Bone Miner Res. 2014;29(3):581-9.

14. Curtis EM, van der Velde R, Moon RJ, van den Bergh JP, Geusens P, de Vries F, van Staa TP, Cooper C, Harvey NC. Epidemiology of fractures in the United Kingdom 1988-2012: variation with age, sex, geography, ethnicity and socioeconomic status. Bone. 2016:87:19-26.

15. Chehade MJ, Carbone T, Awwad D, Taylor A, Wildenauer C, Ramasamy B, McGee M. Influence of fracture stability on early patient mortality and reoperation after Pertrochanteric and intertrochanteric hip fractures. J Orthop Trauma. 2015;29(12):538-43.

16. Cornwall R, Gilbert MS, Koval KJ, Strauss E, Siu AL. Functional outcomes and mortality vary among different types of hip fractures: a function of patient characteristics. Clin Orthop Relat Res. 2004:425:64-71.

17. Lakstein D, Hendel D, Haimovich Y, Feldbrin Z. Changes in the pattern of fractures of the hip in patients 60 years of age and older between 2001 and 2010: a radiological review. Bone Joint J. 2013;95-B(9):1250-4.

18. Martínez AA, Cuenca J, Panisello JJ, Herrera A, Tabuenca A, Canales V. Changes in the morphology of hip fractures within a 10-year period. J Bone Miner Metab. 2001;19(6):378-81.

19. Annual report of RIKSHÖFT, the Swedish National Registry of hip fracture patient care, 2016. Swedish. http://rikshoft.se/wp-content/uploads/2013/07/ rikshoft_rapport2016.pdf. Accessed 1 Mar 2018.

\section{Publisher's Note}

Springer Nature remains neutral with regard to jurisdictional claims in published maps and institutional affiliations. 